\title{
Exposing Pathological Sensory Predictions in Tinnitus Using Auditory Intensity Deviant Evoked Responses
}

\author{
William Sedley, ${ }^{1}$ Kai Alter, ${ }^{1}$ Phillip E. Gander, ${ }^{2}$ Joel Berger, ${ }^{2}$ and $\odot$ Timothy D. Griffiths ${ }^{1,2}$ \\ ${ }^{1}$ Newcastle University Medical School, Newcastle upon Tyne NE2 4HH, United Kingdom, and ${ }^{2}$ Human Brain Research Laboratory, University of Iowa, \\ Hospitals and Clinics, Iowa City, Iowa 52242
}

We tested the popular, unproven theory that tinnitus is caused by resetting of auditory predictions toward a persistent low-intensity sound. Electroencephalographic mismatch negativity responses, which quantify the violation of sensory predictions, to unattended tinnitus-like sounds were greater in response to upward than downward intensity deviants in 26 unselected chronic tinnitus subjects with normal to severely impaired hearing, and in 15 acute tinnitus subjects, but not in 26 hearing and age-matched controls $(p<0.001$, receiver operator characteristic, area under the curve, 0.77 ), or in 20 healthy and hearing-impaired controls presented with simulated tinnitus. The findings support a prediction resetting model of tinnitus generation, and may form the basis of a convenient tinnitus biomarker, which we name Intensity Mismatch Asymmetry, which is usable across species, is quick and tolerable, and requires no training.

Key words: biomarker; electroencephalography; mismatch negativity; phantom perception; predictive coding; tinnitus

\section{Significance Statement}

In current models, perception is based around the generation of internal predictions of the environment, which are tested and updated using evidence from the senses. Here, we test the theory that auditory phantom perception (tinnitus) occurs when a default auditory prediction is formed to explain spontaneous activity in the subcortical pathway, rather than ignoring it as noise. We find that chronic tinnitus patients show an abnormal pattern of evoked responses to unexpectedly loud and quiet sounds that both supports this hypothesis and provides fairly accurate classification of tinnitus status at the individual subject level. This approach to objectively demonstrating the predictions underlying pathological perceptual states may also have a much wider utility, for instance, in chronic pain.

\section{Introduction}

Tinnitus, the persistent perception of an illusory sound, affects $13 \%$, and significantly impairs the quality of life of $2 \%$, of the population (Shargorodsky et al., 2010). The search for effective treatments is greatly hampered by limited understanding of its mechanisms. Its major risk factor is hearing loss, which leads to increased central gain (i.e., increased firing rate and/or synchrony

Received June 6, 2019; revised Sept. 8, 2019; accepted Oct. 4, 2019.

Author contributions: W.S., K.A., and T.D.G. designed research; W.S. performed research; W.S., K.A., P.E.G., J.B., and T.D.G. analyzed data; W.S. wrote the first draft of the paper; W.S., K.A., P.E.G., J.B., and T.D.G. edited the paper; W.S. wrote the paper.

This work was supported by the Academy of Medical Sciences, United Kingdom. W.S. received salary funding from the National Institute for Health Research, United Kingdom. T.D.G. was supported by The Wellcome Trust, United Kingdom.

The authors declare no competing financial interests.

Correspondence should be addressed to William Sedley at william.sedley@newcastle.ac.uk.

https://doi.org/10.1523/JNEUROSCI.1308-19.2019

Copyright $\odot 2019$ Sedley et al.

This is an open-access article distributed under the terms of the Creative Commons Attribution License Creative Commons Attribution 4.0 International, which permits unrestricted use, distribution and reproduction in any medium provided that the original work is properly attributed. in for a given input) (Gold and Bajo, 2014), although a review of current evidence suggests that these changes may be contributory to tinnitus, but not sufficient to cause it (Sedley, 2019), while other evidence suggests that gain increases may be irrelevant, or even protective, with respect to tinnitus (Rüttiger et al., 2013; Singer et al., 2013; Hofmeier et al., 2018), and the presence or absence of hyperacusis can confound results (Gu et al., 2010; Möhrle et al., 2019). We have recently proposed a theory of tinnitus causation, which shares some features with an earlier theory (De Ridder et al., 2014a), in which a crucial process is the learning of a default "tinnitus prediction" by higher perceptual centers (Sedley et al., 2016). Specifically, we suggested that the origin of the tinnitus signal is spontaneous firing in the ascending auditory pathway, but that this is usually successfully ignored as irrelevant noise. Furthermore, we proposed that, once the brain has recognized the tinnitus signal as a sound source, it forms a default prediction of that sound continuing, which prevents the spontaneous activity being ignored as noise, and that prediction ensures the persistence of tinnitus once present for a sufficient length of time. Similar predictive coding (Rao and Ballard, 1999; Friston 


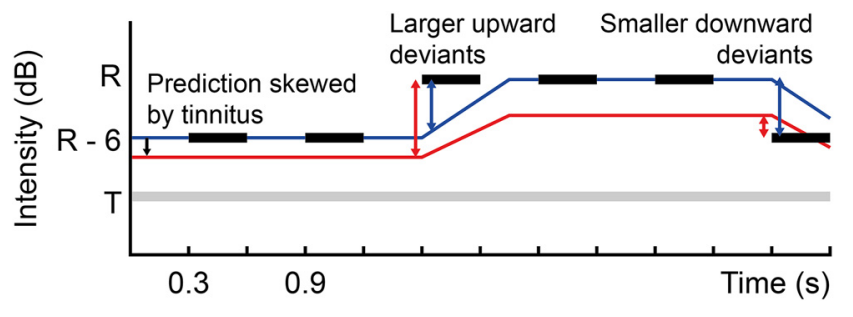

Figure 1. Experimental hypothesis and paradigm. The paradigm is a roving MMN paradigm, with $300 \mathrm{~ms}$ pure tones (black bars) interspersed with $300 \mathrm{~ms}$ intervals. Stimulus frequency is matched to within or adjacent to the individual tinnitus frequency band. Intensity is the roved parameter, between 0 and $-6 \mathrm{~dB}$ relative to an individualized reference intensity (R). We hypothesized that control subjects would make optimal predictions of upcoming stimuli (blue line) based on recent stimulus history, the presence of a tinnitus prediction ( $T$ and gray line) would result in tinnitus subjects forming an intermediate prediction (red line) between the optimal stimulus-based prediction and the tinnitus prediction. This would result in an asymmetry between $M M N$ responses to upward and downward intensity deviants in tinnitus subjects (red arrows) compared with controls (blue arrows). Many aspects of stimulus sequences are predicted, but illustrated predictions here refer only to the intensity of the next upcoming stimulus. Because control subjects' default prediction is of no sound at all, rather than an auditory percept (e.g., tinnitus) but with zero intensity, this does not impact the predicted intensity of upcoming stimuli.

and Kiebel, 2009) models have been proposed for many perceptual disorders (Edwards et al., 2012; Adams et al., 2013; Kumar et al., 2014), but actually demonstrating the aberrant predictions themselves is a much greater challenge; hence, these models rely on circumstantial evidence. The present work aimed to search for evidence of the existence of a default auditory prediction that might underpin chronic tinnitus. We focused on the mismatch negativity (MMN) (Näätänen and Alho, 1995; Näätänen et al., 2007) evoked response, which occurs across many sensory modalities in response to stimuli that differ (e.g., in frequency or intensity) from a series of preceding stimuli. Moreover, MMN magnitude quantitatively indicates the extent to which a particular stimulus violates a prior prediction of what that stimulus will be (Garrido et al., 2013), making it a useful tool for inferring the content of sensory predictions. In this study, we compared MMN responses, obtained from a roving oddball paradigm (Garrido et al., 2008) featuring pure tones close to the tinnitus frequency, to upward and downward intensity deviants to expose alterations of auditory predictions that might be associated with tinnitus (Fig. 1). Specifically, because tinnitus is a quieter sound than those used in the experiment, we hypothesized that the tinnitus prediction should skew predictions of intensity downward, meaning that downward intensity deviants should produce smaller MMN responses, and upward intensity deviants produce larger responses, compared with matched controls. Although there have been numerous MMN studies in tinnitus (Weisz et al., 2004; Holdefer et al., 2013; Mahmoudian et al., 2013; El-Minawi et al., 2018; Mohebbi et al., 2019a), the present study differs importantly in that it both targets the tinnitus frequency and features deviants in intensity, and thus is uniquely able to address this hypothesis. Our results showed a striking asymmetry of MMN responses of exactly the type predicted, compared with age- and hearing-matched controls. This supports the prediction hypothesis of tinnitus (though other interpretations are possible), and can classify individual subjects' tinnitus status with a fair degree of accuracy. The work thus introduces a new potential tinnitus biomarker for further human and animal work.

\section{Materials and Methods}

Subjects. Unselected chronic tinnitus subjects $(n=26)$ were recruited from local research volunteer mailing lists, with the only inclusion crite-
Table 1. Subject, tinnitus, and stimulus characteristics ${ }^{a}$

\begin{tabular}{lcllll}
\hline & Chronic T & Control & $p$ (T vs C) & Acute T & Simulated T \\
\hline Demographics & & & & & \\
Age & $55.4(13.6)$ & $59.7(15.3)$ & 0.31 & $53.8(12.5)$ & $45.0(19.1)$ \\
Sex & $13 \mathrm{~F} / 13 \mathrm{M}$ & $19 \mathrm{~F} / 7 \mathrm{M}$ & $0.014^{*}$ & $6 \mathrm{~F} / 9 \mathrm{M}$ & $10 \mathrm{~F} / 10 \mathrm{M}$ \\
Tinnitus characteristics & & & & & \\
Duration & $15.5(16.7)$ years & - & - & $4.2(1.7)$ weeks & - \\
THI & $31(28.1)$ & - & - & $27(23.2)$ & - \\
T ear & $11 / 15 / 0 \mathrm{~L} / \mathrm{C} / \mathrm{R}$ & - & - & $4 / 8 / 3 \mathrm{~L} / \mathrm{C} / \mathrm{R}$ & - \\
T character & $13 / 13 \mathrm{~T} / \mathrm{N}$ & - & - & $9 / 6 \mathrm{~T} / \mathrm{N}$ & - \\
VAS loudness & $5.0(2.1)$ & - & - & $4.8(1.9)$ & - \\
VAS distress & $4.8(2.9)$ & - & - & $4.8(2.8)$ & - \\
\% awareness & $55.2(34.3)$ & - & - & $44.6(23.7)$ & - \\
T match CF (Hz) & $6777(2009)$ & - & - & $7047(2536)$ & - \\
T match BW (oct) & $0.25(0.25)$ & - & - & $0.17(0.20)$ & - \\
Stimulus and hearing & & & & & - \\
$\quad$ characteristics & & & & & - \\
Center F (Hz) & $7709(2706)$ & & - & $7582(2517)$ & $7164(2861)$ \\
Edge F (Hz) & $5901(1948)$ & & - & $6028(2418)$ & - \\
Edge F to center F (oct) & $0.37(0.27)$ & & - & $0.39(0.27)$ & - \\
Edge F to match & $0.078(0.27)$ & - & - & $0.18(0.18)$ & - \\
$\quad$ lower bound (oct) & & & & & - \\
Thresh center (dB) & $44.8(26.1)$ & $37.9(21.1)$ & 0.19 & $34.3(20.6)$ & $20.3(19.6)$ \\
Thresh edge (dB) & $40.3(21.1)$ & $35.4(17.0)$ & 0.25 & $31.6(22.7)$ & - \\
SPL center (dB) & $78.1(18.4)$ & $78.2(11.2)$ & 0.99 & $79.5(13.0)$ & $79.0(14.1)$ \\
SPL edge (dB) & $72.8(16.4)$ & $78.9(9.68)$ & $0.043^{*}$ & $76.3(12.6)$ & - \\
SL center (dB) & $33.3(23.3)$ & $40.2(20.6)$ & 0.15 & $45.2(23.6)$ & $58.7(20.6)$ \\
SL edge (dB) & $32.5(20.6)$ & $43.4(18.3)$ & $0.012^{*}$ & $44.7(26.8)$ & - \\
\hline
\end{tabular}

${ }^{a}$ Values inside and outside parentheses indicate mean and SD, respectively, unless otherwise indicated. T, Tinnitus; C, control; VAS, visual analog scale; $C F$, center frequency; BW, bandwidth; 0ct, octaves; Thresh, hearing threshold (at specified frequency); SPL, sound pressure level (of stimulus); $S L$, sensation level (of stimulus); $L / C / R$, left (predominant)/center/right (predominant); $T / N$, tonal/narrowband noise; - , not applicable to particular group. ${ }^{*} p<0.05$.

ria being age 18 or over, persistent tinnitus for longer than 6 months, ability to perform experiments, and absence of structural brain pathology or profound hearing loss in the tinnitus ear(s). Nontinnitus controls (matched $n=26$, and simulated tinnitus $n=20$ ) were recruited from the same lists and subjected to pure-tone audiometry, with the best matches being invited to take part in the full study on a separate occasion. Acute tinnitus subjects $(n=15)$ were recruited via paid advertising on an Internet search engine, with the same inclusion criteria as for chronic subjects, but with tinnitus duration of $<6$ weeks. Group sizes were chosen as the minimum necessary to give a high chance of demonstrating the predicted effects. Subject characteristics can be found in Table 1 of the main text. No significant differences in hearing thresholds (Fig. 2) at any frequency were present between chronic tinnitus subjects and matched controls, except at 0.5 and $1 \mathrm{kHz}$ in the right ear, which were remote from the stimulus and tinnitus frequencies $(p<0.05)$.

Recruitment and data collection occurred between November 2017 and October 2018, with additional simulated tinnitus data being collected in August 2019. The study was given a favorable opinion by the Newcastle University Research Ethics Committee, and all participants provided written informed consent according to the Declaration of Helsinki.

Clinical and psychophysical assessment. All research activity occurred within the Institute of Neuroscience, Newcastle University. Subjects completed a short questionnaire covering demographic details, health conditions, and medications. Tinnitus subjects also indicated the duration, character, and laterality of their tinnitus, along with visual analog scale ratings of their average and current tinnitus loudness, average annoyance, and completed the Tinnitus Handicap Inventory (THI) (Newman et al., 1996). All subjects underwent pure tone audiometry at octave intervals from 0.25 to $8 \mathrm{kHz}$, with the addition of $6 \mathrm{kHz}$.

Tinnitus subjects performed five rounds of tinnitus matching, using custom-made tools in MATLAB (The MathWorks), based on interactively tuning narrowband noise with a Hanning-shaped spectrum in terms of its center frequency, bandwidth, intensity, and laterality balance. Each round used random starting parameters for visual analog scale and bandwidth. Subjects were allowed to discard matches they regarded 
as suboptimal, and the mean visual analog scale and bandwidth across remaining matches were used as a starting point for stimulus generation. Control subjects used their matched tinnitus-subject's data for stimulus generation.

For each subject, two pure tone stimuli of different frequencies were created: one at the tinnitus match center and one at the lower edge of the Hanning passband. Tinnitus subjects had one opportunity to tune the frequency of these until perceived as in the center of the tinnitus frequency band ("center"), and the other as close as possible to the tinnitus frequency while being distinctly lower in frequency ("edge"). The rationale for using these frequencies was to test whether any tinnitusrelated effects were tightly locked to the tinnitus frequency, and whether precise tinnitus frequency matching would be required to observe these effects. All subjects then iteratively tuned the following parameters in sequence, until a full round passed with no further changes: laterality balance (center then edge), balancing the subjective loudness of both frequencies, and tuning overall intensity of both frequencies. Control subjects were allocated the stimulus frequencies of the tinnitus subject with the closest audiometric thresholds in stimulus ear/frequency, and were asked to make stimuli as loud as possible without resulting in even minor discomfort, or producing distortions from the headphones. The additional constraint for tinnitus subjects was that tinnitus must remain audible in the gaps between stimuli (i.e., tinnitus not be totally attenuated by residual inhibition).

Experimental design. In a soundproof room, 64 channel EEG was recorded from participants, using an Activetwo system (Biosemi), while they were passively presented with the experimental stimuli through Sennheiser HD 380 pro headphones, and watched a silent subtitled movie. Electrode offset (equivalent to impedance) was kept within manufacturer-recommended limits of $\pm 40 \mathrm{mV}$.

The paradigm was a roving MMN paradigm (Garrido et al., 2009), in which $300 \mathrm{~ms}$ pure tones (with $10 \mathrm{~ms}$ onset/offset ramps) were presented isochronously with a stimulus onset asynchrony of $600 \mathrm{~ms}$. Stimuli were presented to the tinnitus ear if entirely or mainly unilateral (including to the matched control), and bilaterally in other cases. The roved parameter was stimulus intensity, which randomly alternated between 0 and $-6 \mathrm{~dB}$ (relative to the subject-calibrated intensity) every four to eight stimuli. One block of the experiment comprised 21 such intensity changes, and a total of 50 blocks were presented, alternating between the center and edge frequency. A control deviant condition was superimposed on these sequences, whereby 1 in 10 stimuli (1/6 probability, after minimum separation of four stimuli) were duration deviants of $150 \mathrm{~ms}$.

Simulated tinnitus subjects were simultaneously presented with ongoing narrowband noise on alternate blocks ("tinnitus on" condition). This noise had the spectrum of the tinnitus match of the acute or chronic tinnitus subject with the closest hearing thresholds at frequencies adjacent to their tinnitus frequency. To prevent the nonrepresentative scenario of the stimuli and "tinnitus" being perceptually identical, the bandwidth for simulated pure tone tinnitus was set to $1 / 40$ octave. Intensity of the noise stimulus was set at the tinnitus match intensity initially, and the subject was asked to adjust the intensity, if necessary, to ensure it was loud enough to be audible over quiet speech, and quiet enough to not prevent a normal volume conversation. Such adjustments were not required in most cases.

EEG data processing. Data analysis was performed in MATLAB, using the FieldTrip toolbox (Oostenveld et al., 2011). EEG data were recorded at $1024 \mathrm{~Hz}$, downsampled to $256 \mathrm{~Hz}$, and high-pass filtered from $0.3 \mathrm{~Hz}$. Data were rereferenced to combined P9/P10, roughly corresponding to M1 and M2 locations. Bad channels were identified visually and reconstructed by interpolation. Data were epoched between -0.5 and $1 \mathrm{~s}$ peristimulus time, with demeaning and detrending. Epochs with grossly outlying maximum amplitudes, based on visual inspection, were excluded, followed by removal of ocular and muscle artifacts using independent component analysis. A mean of 23 components was removed per subject, with no significant difference in the number of components removed between tinnitus and control groups. Epochs were baseline corrected to $-100-0 \mathrm{~ms}$ peristimulus time, and each epoch was summarized by four values derived from its (normalized within channel) time series: largest absolute amplitude in any channel at any time point; largest mean absolute deviation across channels at any time point; largest mean absolute amplitude across time at any channel; and largest mean absolute amplitude across time and channels. Histograms were plotted of the four values, and thresholds for trial rejection specified manually based on the point where the upper tail deviates from a normal distribution. Epochs were rejected if any of their four values exceeded its threshold, and $\sim 10 \%$ of trials were rejected for each subject. Visual inspection of a subset of epoch waveforms confirmed that this method removed bad epochs successfully. Surviving epochs were averaged within their respective stimulus conditions, followed by low-pass filtering at $35 \mathrm{~Hz}$.

Based on evoked peak topographies observed in pilot experiments, $\mathrm{FCz}$ was chosen as the sole channel from which to present time-domain data, and three time windows were determined that maximally captured the three deflections characterizing the evoked response. The mean evoked potential within each time window was taken as the basis for statistical analysis.

Because we had no hypothesis about MMN latency, the duration of MMN responses was relatively long, and there were no clear differences in MMN latency, we did not subject MMN latency to any formal analysis. The primary outcome measure was MMN amplitude, with MMNtimeframe response magnitudes to standards, and N1 and P50 magnitudes as secondary outcome measures.

Statistical analysis. Statistical analysis was performed in MATLAB. On account of Lillefort's test not indicating more datasets deviating from a normal distribution than expected by chance, ANOVA was used as the basis for statistical analysis. For comparison of chronic tinnitus subjects and controls, a three-way ANOVA with full interaction terms was applied, with group (tinnitus or control), frequency (edge or center), and intensity (low or high) as the factors of interest. As the acute tinnitus group was not matched to a control group, it was subject to a two-way ANOVA, with interaction term, with frequency and intensity as the factors of interest. The simulated tinnitus group was subject to a two-way ANOVA, with interaction term, with state ("tinnitus" on or off) and intensity as the factors of interest. Each ANOVA was performed separately on standards and deviants (deviants minus standards). The receiver-operator characteristic (ROC) curve was generated using standard MATLAB functions. 
A

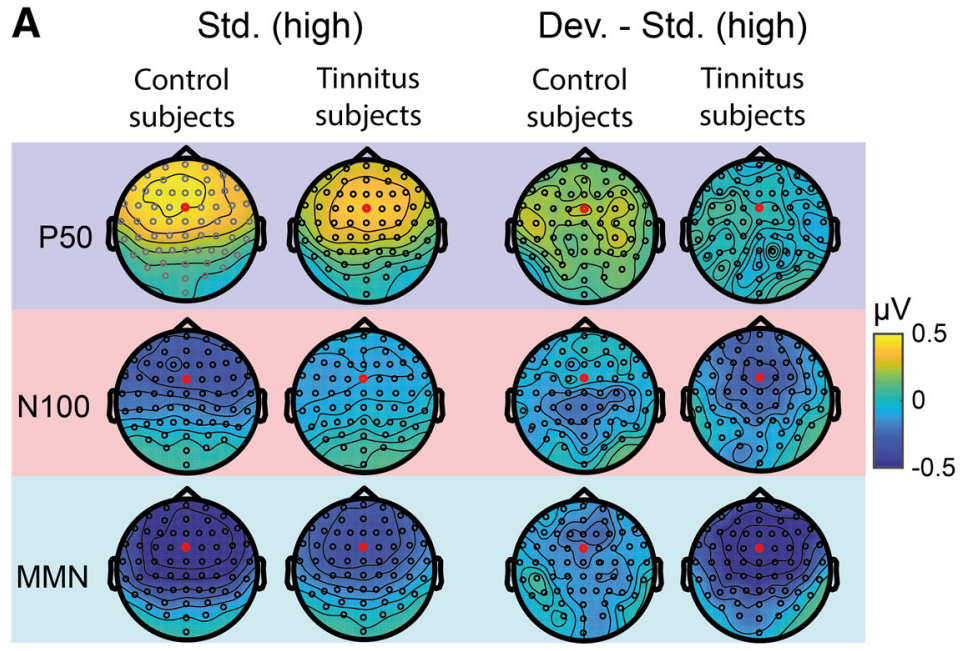

B
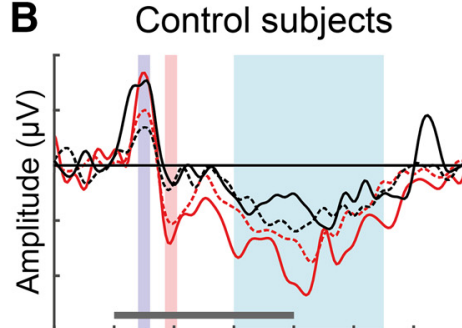

Tinnitus subjects

Edge freq., Std. ----- Centre freq., Std.
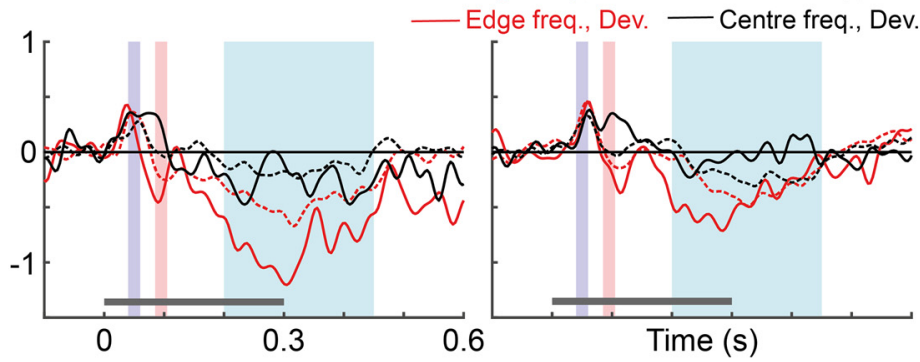

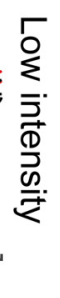

Figure 3. Spatiotemporal distribution of evoked responses. $A$, Group mean scalp topographies within color-coded time windows capturing the three dominant waveforms. Illustrative responses are shown for the high-intensity standards, and upward deviant minus high standard responses, only. Std., Standard.; Dev., deviant. $\boldsymbol{B}$, Evoked waveforms from the FCz electrode, highlighted in red in $\boldsymbol{A}$, to both standard (dashed) and intensity deviant (solid) stimuli at the tinnitus center (black) and edge (red) frequencies. Gray horizontal bars represent stimulus presentation. Colored vertical bars represent the color-coded time windows corresponding to $\mathrm{P} 50, \mathrm{~N} 100$, and $\mathrm{MMN}$, as shown in $\boldsymbol{A}$, and forming the basis of statistical analyses.

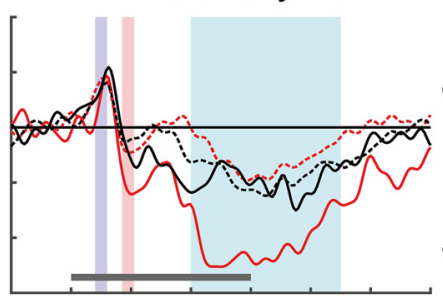

the 6 tinnitus subjects with the lowest stimulus intensities (in $\mathrm{dB}$ SL) at the edge frequency; this balanced the group means for the edge frequency stimulus intensity (41.0 vs $42.7 \mathrm{~dB}$ SL, $p=0.75$ ) and increased the statistical significance of the main finding (discussed in its respective section) from $p=0.0009$ to $p=0.0001$. The inclusion of unselected tinnitus subjects, including those with severe high-frequency hearing loss, older volunteers, and both tonal and narrowband noise types of tinnitus, potentially may have added noise and variance to the data, but we considered this inclusivity important to prove the applicability of any findings to the broader tinnitus population as opposed to a particular subset.

\section{Spatiotemporal organization of stimulus response}

In a roving MMN paradigm (Fig. 1), with isochronous $300 \mathrm{~ms}$ pure tones matched to either the center frequency or lower edge (subjectively defined as "just outside" the tinnitus sound) of the tinnitus frequency band as the stimuli, and stimulus intensity as the roved parameter, the event-related potential was characterized (Fig. 3) by approximately equally sized P50 and N100 responses, and a prolonged late negative potential, peaking at $200-$ $450 \mathrm{~ms}$ in keeping with the timeframe of $M M N$. This is long for MMN in general, but we note that the one study to examine intensity deviants in tinnitus (Mahmoudian et al., 2013) showed later responses to these than other deviants, in keeping with what we observed here. Furthermore, it is recognized that smaller perceptual changes are associated with later MMN responses (Näätänen and Alho, 1995).

\section{Results}

\section{Subject characteristics}

Subject groups comprised 26 unselected chronic tinnitus subjects, 26 age- and hearing-matched controls, 15 acute tinnitus subjects, and 20 healthy controls studied with and without the simultaneous presentation of simulated tinnitus based on tinnitus subjects' psychophysical tinnitus matches. Their characteristics, along with their individual tinnitus matches and derived stimulus parameters, are summarized in Table 1 . Due partly to prioritizing audiometric matches at the stimulus frequencies, there were significant differences between chronic tinnitus and matched control groups in sex and stimulus intensity. The latter may have reflected the matching procedure or (appropriate) compensation for hyperacusis in the tinnitus group. Stimulus loudness at the tinnitus edge frequency was, on average, higher in the control than chronic tinnitus group (Table 1), but overlap between groups was high. To ensure that this did not lead to spurious results, we repeated the primary analysis after excluding
Early auditory evoked potentials (P50 and N100) are unaffected by tinnitus

There were no differences in standard or deviant P50 responses related to subject group, stimulus frequency, or stimulus intensity. In a three-way ANOVA (subject group, stimulus frequency, and stimulus intensity), N100 response magnitudes to standard stimuli showed a main effect of larger responses to high-intensity stimuli $(p<0.05)$, which was an expected and trivial finding. An equivalent analysis of deviant-minus-standard responses showed a main effect of larger responses to the tinnitus edge (lower) than tinnitus center (higher) frequency $(p<0.005)$. The lack of differences between tinnitus and control groups in these early responses makes simple acoustic differences in stimuli, such as loudness, an unlikely explanation for the tinnitus-related changes described below.

\section{Tinnitus-irrelevant duration deviants}

To exclude broad differences in predictive processes not specifically related to tinnitus or the underlying hypothesis, we incor- 

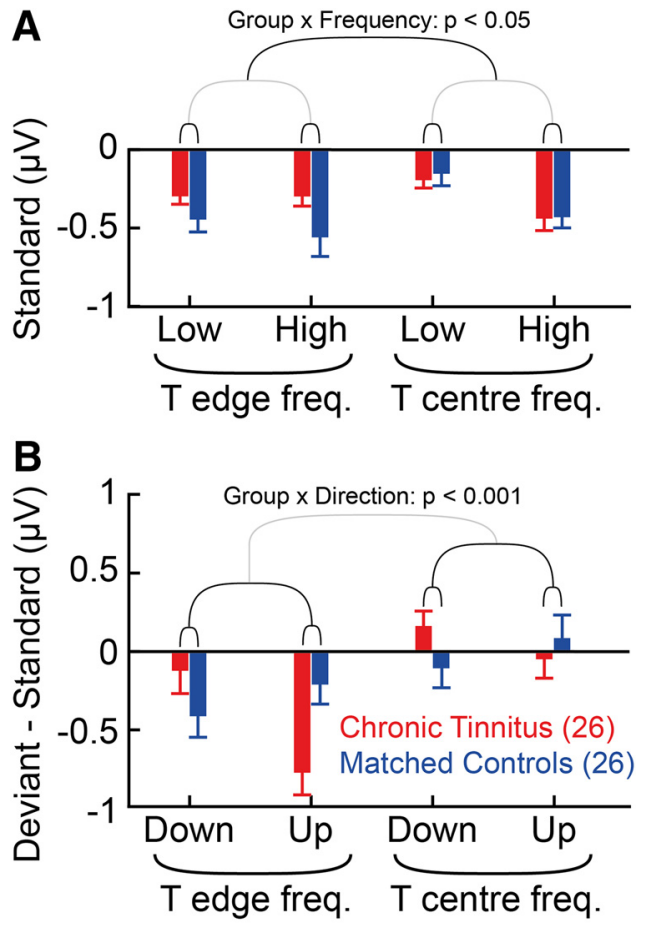

Stimulus intensity/frequency

Figure 4. MMN amplitudes in chronic tinnitus and control subjects. Error bars indicate group mean \pm SE. Color coding represents subject group: Red represents chronic tinnitus. Blue represents matched controls. $A, M M N$ timeframe responses to standard stimuli. $\boldsymbol{B}$, MMN responses to deviant minus standard responses. Significant differences relevant to tinnitus status are indicated by $p$ values and nested brackets. Black brackets represent variables, or interactions, associated with significant effects based on ANOVA. Gray brackets represent variables not significantly contributing to effects. The core finding was a significant $(p<0.001)$ group (chronic tinnitus vs control) $\times$ direction (upward vs downward) interaction in deviant MMN responses.

porated occasional shorter $150 \mathrm{~ms}$ stimuli to serve as duration deviants. Duration deviants, in the absence of intensity changes, elicited clear MMN responses, which showed no significant differences on account of stimulus frequency or intensity, or subject group.

\section{Late (MMN timeframe) responses to standard stimuli show} small differences due to tinnitus

Standard responses in the MMN time window showed a main effect of being larger for high- as opposed to low-intensity stimuli $(p<0.005)$, which was expected. As shown in Figure $4 A$, there was a group $\times$ frequency interaction $(p<0.05)$, whereby the control group, but not the tinnitus group, had larger responses to the lower (edge) frequency standards.

\section{Asymmetry of MMN responses to intensity deviants differentiates tinnitus subjects from controls}

There was a main effect of larger deviant minus standard responses to the lower (edge) than higher (center) frequency $(p<$ 0.0001 ). This mirrored the equivalent difference seen in the N1 responses to deviants, and is likely to be for the same reason. As shown in Figure $4 B$, our principal finding was in line with our hypothesis, in that there was a group $\times$ direction interaction $(p<0.001)$, whereby tinnitus subjects had larger responses to upward deviants (minus standards), whereas matched controls had larger responses to downward deviants (minus standards). The effect, which we term Intensity Mismatch Asymmetry
(IMA), applied to both tinnitus center and tinnitus edge frequencies; but in keeping with the main effect of frequency, the absolute effect appeared larger at the edge frequency, although the contribution of frequency to this interaction was not statistically significant. Repeating the analysis after excluding the 6 tinnitus subjects with the least intense edge frequency stimuli (to balance mean intensity with the control group) showed the same finding, but with the greater level of statistical significance of $p=0.0001$.

To assess whether IMA can serve as a biomarker for tinnitus, we used the simple metric (averaged across stimulus frequencies) of upward deviants (minus standards) minus downward deviants (minus standards). Figure 5 shows the ROC curve for this metric, which results in an area under the curve of 0.77 , indicating the favorable end of "fair" diagnostic accuracy. We aimed to avoid the use of a more complicated classifier metric, which might have produced greater accuracy, to constrain our findings to those that might be ported directly to animal studies. No significant linear correlations were observed between this metric and either THI or visual analog scale loudness score.

\section{Tinnitus-related MMN changes are present in the acute, as well as chronic, stages}

We have previously hypothesized that there is a window of reversibility following initial tinnitus onset, before the tinnitus prediction becomes pervasive, although the length of this window would be unclear, potentially ranging from a scale of days to months. In a two-way ANOVA (intensity and frequency), the group of 15 subjects with new-onset tinnitus (usually within the past 3-4 weeks) showed a main effect of upward intensity deviants yielding larger MMN responses than downward deviants $(p<0.05)$.

\section{Absence of equivalent changes in simulated tinnitus}

The above finding of IMA could theoretically be for the reason hypothesized, that the aberrant prediction responsible for tinnitus skews sensory predictions, or for the more trivial, but still diagnostically useful, reason that the presence of any quiet continuous sound alongside the stimuli skews predictions toward that quiet intensity. To distinguish these possibilities, we conducted the same experiment in 20 healthy controls, with and without hearing loss, with half the blocks containing the addition of simulated tinnitus based on tinnitus subjects' matching data. To maintain a sufficient number of trials, only stimuli at the tinnitus center frequency were used. No significant differences were found between the "tinnitus on" and "tinnitus off" state; in a two-way ANOVA featuring deviant direction (up or down) and state (on or off), the $p$ values for main effects of state and state $X$ direction interaction were 0.81 and 0.77 , respectively.

\section{Discussion}

IMA differentiates tinnitus subjects from controls

We tested the hypothesis that development of chronic tinnitus requires formation of a pervasive "default" prediction of a (usually quiet) constant sound within a specific frequency band, and that this prediction favors perceptual recognition of tonotopically specific spontaneous firing in the auditory pathway as a real sound (i.e., tinnitus) rather than ignoring as noise (Sedley et al., 2016). Processing of auditory stimuli within or close to the relevant frequency band might be altered by skewing of all predictions toward the characteristics of the default prediction (Fig. 1). We hypothesized that these skewed predictions would be detectable in MMN responses to intensity deviants around the tinnitus frequency; because downward deviations in intensity involve 


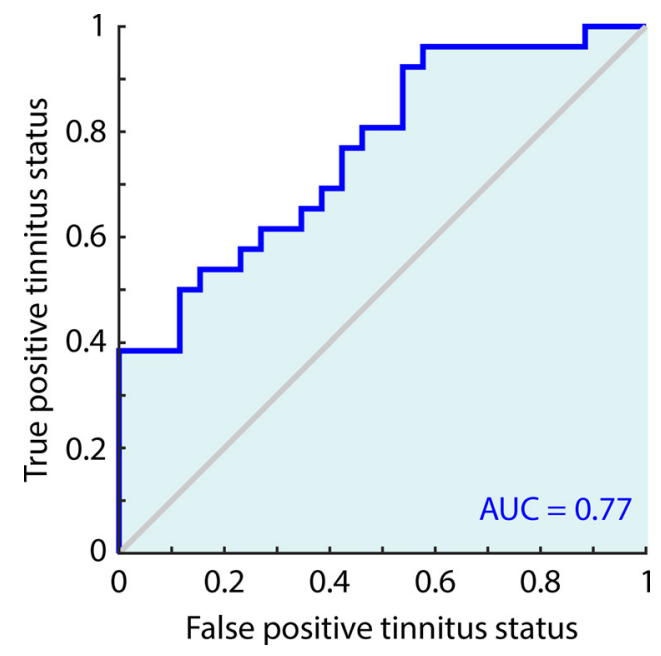

Figure 5. ROC curve for classification of subjects as chronic tinnitus or matched control.

stimuli becoming quieter, therefore, more similar to the default prediction, tinnitus subjects would show reduced response magnitudes. Conversely, upward deviations in intensity involve stimuli becoming louder; hence, more distant from the default prediction, tinnitus subjects would show increased response magnitudes. Thus, we hypothesized that contrast between upward and downward intensity deviants might serve as an objective marker of tinnitus, and our results support this hypothesis in both the acute (at $\sim 4$ weeks from onset) and chronic stages of tinnitus.

\section{The IMA effect reflects tinnitus specifically}

In theory, differences in MMN responses might occur simply because of an ongoing sound filling in the interstimulus gaps, in which case IMA would be an epiphenomenon of tinnitus, rather than a causative factor. To differentiate these possibilities, we studied 20 nontinnitus controls with and without the simultaneous presentation of narrowband noise derived from subjects' tinnitus matches. Short-term simulated tinnitus should not alter default predictions of the kind hypothesized to underlie tinnitus because: (1) we only presented it for $\sim 60 \mathrm{~s}$ at a time, which would not be a long enough timescale to form pervasive default predictions; and (2) not everybody would necessarily change their default prediction, even after a sufficiently long duration.

The addition of simulated tinnitus within this second control group did not produce any appreciable change in MMN responses, suggesting a specific role in tinnitus.

\section{Mechanisms potentially underlying IMA}

Auditory MMN is generated by a bilateral network of primary and nonprimary auditory cortex and inferior frontal gyrus (Garrido et al., 2008). Reciprocal interaction between these centers is argued to comprise the bottom-up propagation of prediction errors, which signal discordance between prior prediction and sensory input, and the top-down updating of sensory predictions in light of this new evidence (Garrido et al., 2008, 2009), although other explanations include sensory memory, local adaptation to a stimulus, and change detection (Garrido et al., 2009). MMN amplitude is also sensitive to higher-level statistical structure in stimulus sequences (Garrido et al., 2013), and therefore also provides a quantitative indication of the improbability of a stimulus based on prior predictions. Ventrolateral PFC (including inferior frontal gyrus) has been argued to form part of a "tinnitus core" network, which also includes auditory, inferior parietal, and parahippocampal cortex (De Ridder et al., 2014b). Parahippocampal cortex has shown altered resting-state activity contralateral to the tinnitus ear (Vanneste et al., 2011) and resting-state fMRI correlation with auditory cortex (Maudoux et al., 2012; Schmidt et al., 2013), and based on its prominent role in auditory memory is a potential source of persistent auditory predictions. While these networks are likely contributors to the IMA effect we observed here, as the present study does not provide source-resolved activity, it does not in itself specify the brain basis of the effect. Future work might address this issue with imaging modalities with higher spatial resolution.

MMN magnitudes might be affected by changes in central gain, including related to hyperacusis, or deficient noise cancellation via frontostriatal gating, which amounts to a gain control mechanism (Leaver et al., 2011; Rauschecker et al., 2015). P50 suppression is often used as a marker cortical input gating (Yadon et al., 2009) and might have been expected to be a sensitive marker of any gating changes if present. However, there were no differences in any evoked response magnitudes to standard stimuli between tinnitus and control groups, suggesting against a straightforward gain or hyperacusis-related explanation. There are, however, more nuanced aspects of gain, such as dynamic range adaptation, and so we cannot altogether rule out changes in gain in the broader sense as a contributory factor.

We attempted to standardize attention by having all subjects watch a subtitled movie they found engaging, and all subjects claimed they were able to largely ignore the auditory stimuli and attend to the movie. However, this was not formally quantified; hence, some differences between groups cannot be ruled out. Similarly, subjects with substantial tinnitus-related distress might attend more to auditory stimuli or perceive intensity increases in a more threatening way. However, we observed no correlation between magnitude of IMA effect and THI score.

\section{Previous MMN studies in tinnitus}

Previous MMN and equivalent studies (Weisz et al., 2004; Holdefer et al., 2013; Mahmoudian et al., 2013; Asadpour et al., 2018; El-Minawi et al., 2018; Mohebbi et al., 2019b) have varied according to the type of deviant, the paradigm used, control matching for hearing loss, and, importantly, whether stimulus frequencies were standardized or targeted to subjects' tinnitus. Studies with nontargeted stimulus frequencies have reported slightly smaller MMN responses to deviants of all types tested (Holdefer et al., 2013; Mahmoudian et al., 2013), and minor differences in P300 oddball responses to auditory and visual stimuli (Asadpour et al., 2018). At the audiometric (not tinnitus) edge frequency, tinnitus patients showed larger MMN responses (in the N1 timeframe) to downward frequency deviants than hearing unmatched controls (Weisz et al., 2004), and unchanged responses one octave lower. Frequency deviants, with the deviant at the tinnitus match frequency, and the control frequency $10 \%$ different, have been found to be increased compared with controls, with partial resolution of the difference following successful tinnitus retraining therapy (El-Minawi et al., 2018). Using standardized stimulus frequencies at $\sim 8 \mathrm{kHz}$ (regardless of tinnitus frequency), smaller MMN responses were observed in tinnitus patients with high levels of distress only (Mohebbi et al., 2019b). These studies set a precedent for there being small differences in sensory, mnemonic, and/or predictive processing relevant to the MMN in tinnitus. Our present study is the first to feature intensity deviants targeted to the tinnitus frequency; and as such, our results show a 
much stronger effect and may provide a way forward for this specific field in tinnitus research.

\section{Potential use as a biomarker}

Successful animal research into tinnitus mechanisms and treatments requires knowing which animals experience tinnitus. $\mathrm{Nu}$ merous methods have been developed to determine this and broadly fall into two categories. Conditioned behavior models (Rüttiger et al., 2003; Brozoski and Bauer, 2016; Pace et al., 2016) are often regarded as the more accurate, and require lengthy prior training of animals to perform or refrain from certain behaviors, such as licking, during the presence of an ongoing sound. Automatic response methods (Turner et al., 2006; Lobarinas et al., 2013) have the advantage of requiring no training, and exploit involuntary responses, such as the acoustic startle response, in conjunction with stimuli related to the possible tinnitus (e.g., a short gap in an ongoing pure tone) to modify this depending on tinnitus status, but are subject to caveats and controversies (Campolo et al., 2013; Lobarinas et al., 2013), and show inconsistent replicability in humans (Fournier and Hébert, 2013; Shadwick and Sun, 2014; Boyen et al., 2015). The two types of approach have shown limited correlation with each other, and with the presence or absence of an auditory insult potentially sufficient to induce tinnitus (Turner et al., 2006). However, because there is no gold standard in animals against which to test the sensitivity and specificity of a diagnostic tinnitus test, the performance of these measures remains unquantified. Potential biomarkers derived from human tinnitus studies have mainly focused on whole-brain resting-state imaging of electrical activity (Vanneste et al., 2018) or large-scale correlations in cerebral blood flow (Minami et al., 2018; Zimmerman et al., 2019), but these measures are inherently nontransferrable to animals. The IMA technique, as reported here, has the potential to constitute a diagnostic test that is quantifiable in its diagnostic performance, on account of being developed in humans, applicable across species, free from training requirements, and quick to perform. The presence of the IMA effect, even just outside the tinnitus frequency, suggests that its success does not depend upon highly specific tinnitus matching. Another recent study has had the same aim (Han et al., 2017), based on quantifying the acoustic change complex, an evoked response to a change during a stimulus. It yielded only slightly lower ROC performance, but we note that the study was subject to numerous limitations, including only yielding this result at uncomfortably loud stimulus levels, and excluding subjects who were older or had significant hearing loss. Although there are a number of factors to address in follow-up studies (e.g., tuning curves over stimulus frequency and intensity, optimizing stimulus timing and duration, standardized diagnostic cutoffs), we believe the IMA technique might have the potential to serve as a convenient and robust biomarker for future animal studies of tinnitus.

\section{Parallels with other perceptual disorders}

Predictive coding accounts of perception (Rao and Ballard, 1999; Friston and Kiebel, 2009) are popular in neuroscience, and our predictive coding tinnitus model (Sedley et al., 2016) joins other predictive coding models of tinnitus (De Ridder et al., 2014a; Durai et al., 2018), and other pathological perceptual states, including chronic pain (De Ridder et al., 2014a; Hechler et al., 2016; Geuter et al., 2017; Nir and Yarnitsky, 2015), musical hallucinosis (Kumar et al., 2014), psychosis (Adams et al., 2013), and functional neurological disorder (Edwards et al., 2012). These theoretical models generally lack support by measurement of the pathological predictions themselves. Here, we demonstrate proof of concept that pathological predictions can be measured using cheap, widely available tools. As it shares many parallels with tinnitus (Møller, 1997; De Ridder et al., 2011, 2014a; Rauschecker et al., 2015; Vanneste et al., 2018), chronic pain would be a logical condition to extend this approach to next.

\section{References}

Adams RA, Stephan KE, Brown HR, Frith CD, Friston KJ (2013) The computational anatomy of psychosis. Front Psychiatry 4:47.

Asadpour A, Alavi A, Jahed M, Mahmoudian S (2018) Cognitive memory comparison between tinnitus and normal cases using event-related potentials. Front Integr Neurosci 12:48.

Boyen K, Baskent D, van Dijk P (2015) The gap detection test: can it be used to diagnose tinnitus? Ear Hear 36:e138-e145.

Brozoski TJ, Bauer CA (2016) Animal models of tinnitus. Hear Res 338:88-97.

Campolo J, Lobarinas E, Salvi R (2013) Does tinnitus "fill in" the silent gaps? Noise Health 15:398-405.

De Ridder D, Elgoyhen AB, Romo R, Langguth B (2011) Phantom percepts: tinnitus and pain as persisting aversive memory networks. Proc Natl Acad Sci U S A 108:8075-8080.

De Ridder D, Vanneste S, Freeman W (2014a) The Bayesian brain: phantom percepts resolve sensory uncertainty. Neurosci Biobehav Rev 44:4-15.

De Ridder D, Vanneste S, Weisz N, Londero A, Schlee W, Elgoyhen AB, Langguth B (2014b) An integrative model of auditory phantom perception: tinnitus as a unified percept of interacting separable subnetworks. Neurosci Biobehav Rev 44:16-32.

Durai M, O'Keeffe MG, Searchfield GD (2018) A review of auditory prediction and its potential role in tinnitus perception. J Am Acad Audiol 29: 533-547.

Edwards MJ, Adams RA, Brown H, Pareés I, Friston KJ (2012) A Bayesian account of "hysteria." Brain 135:3495-3512.

El-Minawi MS, Dabbous AO, Hamdy MM, Sheta SM (2018) Do [sic] changes in mismatch negativity after tinnitus retraining therapy using tinnitus pitch as deviant stimulus, reflect subjective improvement in tinnitus handicap? Hear Balance Commun 16:182-196.

Fournier P, Hébert S (2013) Gap detection deficits in humans with tinnitus as assessed with the acoustic startle paradigm: does tinnitus fill in the gap? Hear Res 295:16-23.

Friston K, Kiebel S (2009) Predictive coding under the free-energy principle. Philos Trans R Soc Lond B Biol Sci 364:1211-1221.

Garrido MI, Friston KJ, Kiebel SJ, Stephan KE, Baldeweg T, Kilner JM (2008) The functional anatomy of the MMN: a DCM study of the roving paradigm. Neuroimage 42:936-944.

Garrido MI, Kilner JM, Stephan KE, Friston KJ (2009) The mismatch negativity: a review of underlying mechanisms. Clin Neurophysiol 120:453463.

Garrido MI, Sahani M, Dolan RJ (2013) Outlier responses reflect sensitivity to statistical structure in the human brain. PLoS Comput Biol 9:e1002999.

Geuter S, Boll S, Eippert F, Büchel C (2017) Functional dissociation of stimulus intensity encoding and predictive coding of pain in the insula. Elife 6:e24770.

Gold JR, Bajo VM (2014) Insult-induced adaptive plasticity of the auditory system. Front Neurosci 8:110.

Gu JW, Halpin CF, Nam EC, Levine RA, Melcher JR (2010) Tinnitus, diminished sound-level tolerance, and elevated auditory activity in humans with clinically normal hearing sensitivity. J Neurophysiol 104:3361-3370.

Han JH, Won JY, Hong SK, Kim JH, Kim ES, Kim HJ, Lee HJ (2017) Objective measurement of subjective tinnitus using the acoustic change complex. PLoS One 12:e0188268.

Hechler T, Endres D, Thorwart A (2016) Why harmless sensations might hurt in individuals with chronic pain: about heightened prediction and perception of pain in the mind. Front Psychol 7:1638.

Hofmeier B, Wolpert S, Aldamer ES, Walter M, Thiericke J, Braun C, Zelle D, Rüttiger L, Klose U, Knipper M (2018) Reduced sound-evoked and resting-state BOLD fMRI connectivity in tinnitus. Neuroimage Clin 20: 637-649.

Holdefer L, Oliveira CA, Ramos Venosa A (2013) The mismatch negativity test in ears with and without tinnitus-a path to the objectification of tinnitus abstract. Int Tinnitus J 18:177. 
Kumar S, Sedley W, Barnes GR, Teki S, Friston KJ, Griffiths TD (2014) A brain basis for musical hallucinations. Cortex 52:86-97.

Leaver AM, Renier L, Chevillet MA, Morgan S, Kim HJ, Rauschecker JP (2011) Dysregulation of limbic and auditory networks in tinnitus. Neuron 69:33-43.

Lobarinas E, Hayes SH, Allman BL (2013) The gap-startle paradigm for tinnitus screening in animal models: limitations and optimization. Hear Res 295:150-160.

Mahmoudian S, Farhadi M, Najafi-Koopaie M, Darestani-Farahani E, Mohebbi M, Dengler R, Esser KH, Sadjedi H, Salamat B, Danesh AA, Lenarz $\mathrm{T}$ (2013) Central auditory processing during chronic tinnitus as indexed by topographical maps of the mismatch negativity obtained with the multi-feature paradigm. Brain Res 1527:161-173.

Maudoux A, Lefebvre P, Cabay JE, Demertzi A, Vanhaudenhuyse A, Laureys S, Soddu A (2012) Connectivity graph analysis of the auditory resting state network in tinnitus. Brain Res 1485:10-21.

Minami SB, Oishi N, Watabe T, Uno K, Ogawa K (2018) Auditory related resting state fMRI functional connectivity in tinnitus patients. Otol Neurotol 39:1-5.

Mohebbi M, Daneshi A, Asadpour A, Mohsen S, Farhadi M, Mahmoudian S (2019a) The potential role of auditory prediction error in decompensated tinnitus: an auditory mismatch negativity study. Brain Behav 9:e01242.

Mohebbi M, Daneshi A, Asadpour A, Mohsen S, Farhadi M, Mahmoudian S (2019b) The potential role of auditory prediction error in decompensated tinnitus: an auditory mismatch negativity study. Brain Behav 9:e01242.

Möhrle D, Hofmeier B, Amend M, Wolpert S, Ni K, Bing D, Klose U, Pichler B, Knipper M, Rüttiger L (2019) Enhanced central neural gain compensates acoustic trauma-induced cochlear impairment, but unlikely correlates with tinnitus and hyperacusis. Neuroscience 407:146-169.

Møller AR (1997) Similarities between chronic pain and tinnitus. Am J Otol 18:577-585.

Näätänen R, Alho K (1995) Mismatch negativity: a unique measure of sensory processing in audition. Int J Neurosci 80:317-337.

Näätänen R, Paavilainen P, Rinne T, Alho K (2007) The mismatch negativity (MMN) in basic research of central auditory processing: a review. Clin Neurophysiol 118:2544-2590.

Newman CW, Jacobson GP, Spitzer JB (1996) Development of the tinnitus handicap inventory. Arch Otolaryngol Head Neck Surg 122:143-148.

Nir RR, Yarnitsky D (2015) Conditioned pain modulation. Curr Opin Support Palliat Care 9:131-137.

Oostenveld R, Fries P, Maris E, Schoffelen JM (2011) FieldTrip: open source software for advanced analysis of MEG, EEG, and invasive electrophysiological data. Comput Intell Neurosci 2011:156869.

Pace E, Luo H, Bobian M, Panekkad A, Zhang X, Zhang H, Zhang J (2016) A conditioned behavioral paradigm for assessing onset and lasting tinnitus in rats. PLoS One 11:e0166346.
Rao RP, Ballard DH (1999) Predictive coding in the visual cortex: a functional interpretation of some extra-classical receptive-field effects. Nat Neurosci 2:79-87.

Rauschecker JP, May ES, Maudoux A, Ploner M (2015) Frontostriatal gating of tinnitus and chronic pain. Trends Cogn Sci 19:567-578.

Rüttiger L, Ciuffani J, Zenner HP, Knipper M (2003) A behavioral paradigm to judge acute sodium salicylate-induced sound experience in rats: a new approach for an animal model on tinnitus. Hear Res 180:39-50.

Rüttiger L, Singer W, Panford-Walsh R, Matsumoto M, Lee SC, Zuccotti A, Zimmermann U, Jaumann M, Rohbock K, Xiong H, Knipper M (2013) The reduced cochlear output and the failure to adapt the central auditory response causes tinnitus in noise exposed rats. PLoS One 8:e57247.

Schmidt SA, Akrofi K, Carpenter-Thompson JR, Husain FT (2013) Default mode, dorsal attention and auditory resting state networks exhibit differential functional connectivity in tinnitus and hearing loss. PLoS One 8:e76488.

Sedley W (2019) Tinnitus: does gain explain? Neuroscience 407:213-228.

Sedley W, Friston KJ, Gander PE, Kumar S, Griffiths TD (2016) An integrative tinnitus model based on sensory precision. Trends Neurosci 39:799-812.

Shadwick K, Sun W (2014) Acoustic startle reflex and pre-pulse inhibition in tinnitus patients. J Otol 9:141-145.

Shargorodsky J, Curhan GC, Farwell WR (2010) Prevalence and characteristics of tinnitus among US adults. Am J Med 123:711-718.

Singer W, Zuccotti A, Jaumann M, Lee SC, Panford-Walsh R, Xiong H, Zimmermann U, Franz C, Geisler HS, Köpschall I, Rohbock K, Varakina K, Verpoorten S, Reinbothe T, Schimmang T, Rüttiger L, Knipper M (2013) Noise-induced inner hair cell ribbon loss disturbs central arc mobilization: a novel molecular paradigm for understanding tinnitus. Mol Neurobiol 47:261-279.

Turner JG, Brozoski TJ, Bauer CA, Parrish JL, Myers K, Hughes LF, Caspary DM (2006) Gap detection deficits in rats with tinnitus: a potential novel screening tool. Behav Neurosci 120:188-195.

Vanneste S, Heyning PV, De Ridder D (2011) Contralateral parahippocampal gamma-band activity determines noise-like tinnitus laterality: a region of interest analysis. Neuroscience 199:481-490.

Vanneste S, Song JJ, De Ridder D (2018) Thalamocortical dysrhythmia detected by machine learning. Nat Commun 9:1103.

Weisz N, Voss S, Berg P, Elbert T (2004) Abnormal auditory mismatch response in tinnitus sufferers with high-frequency hearing loss is associated with subjective distress level. BMC Neurosci 5:8.

Yadon CA, Bugg JM, Kisley MA, Davalos DB (2009) P50 sensory gating is related to performance on select tasks of cognitive inhibition. Cogn Affect Behav Neurosci 9:448-458.

Zimmerman BJ, Abraham I, Schmidt SA, Baryshnikov Y, Husain FT (2019) Dissociating tinnitus patients from healthy controls using resting-state cyclicity analysis and clustering. Netw Neurosci 3:67-89. 